\title{
Specific Median Flour Particle Size Distribution of Japanese Common Wheats; Comparison with Chinese Common Wheats
}

\author{
H. NAKAMURA* \\ N.A.R.O. Institute of Crop Science Tsukuba, Ibaraki, Japan \\ (Received 30 June 2014; Accepted 29 October 2014; \\ Communicated by F. Békés)
}

\begin{abstract}
Flour particle size distribution is a major factor affecting the milling behavior in common wheats (Triticum aestivum L.). Also, a previous study has revealed a relationship between flour yield and median flour particle size of Japanese wheats. However, the genetic base of Japanese common wheat is narrow and will need to be broadened. Therefore, in order to help develop the genetic resource for higher flour yields in Japanese wheat breeding, the flour yield of one hundred and seventy Chinese common wheats were investigated in relation to the median flour particle size and the flour particle size distribution patterns as determined by laser diffraction. The results showed significant differences in the median flour particle size and distribution patterns of Chinese wheats and the Japanese wheats that had been analyzed in a previous study. In particular, $45 \%$ of Chinese wheats were categorized as hard wheats, but only $4 \%$ as soft wheats. In contrast, the previously analyzed Japanese wheat proportions were $20 \%$ and $25 \%$, respectively. The results show that analysis of median flour particle size distribution by laser diffraction is a simple but effective tool for measuring and selecting the good flour milling quality in Chinese wheat genetic resources. In addition, the major differences between the Japanese and Chinese wheats highlighted in this study occurred despite the fact that it is well known that Chinese common wheats contributed to modern Japanese wheat varieties. The differences may be explained by either the founder effect or by a selective bottleneck in Japanese wheat genetic resources.
\end{abstract}

Keywords: median flour particle size, soft type, breeding material, bottleneck effect, Chinese common wheat

\section{Introduction}

It is important to develop Japanese udon-noodle wheat varieties with a higher flour yield and enhanced grain quality, in order to satisfy the demands of Japanese milling companies and improve the international competiveness of Japanese wheat. Therefore, an important factor in Japanese wheat breeding programs is to breed udon-wheat lines with an excellent milling quality (Yamashita 1994). It has well known that flour hardness is associated with flour strength, but relatively little information has been published about flour hardness of Japanese wheat varieties (Ikeda 1961; Nakamura et al. 1990). Further-

\footnotetext{
*E-mail: hiro@affrc.go.jp
} 
more, little research has been conducted on simple and reliable assessments of the flour milling quality needed for Japanese udon-noodle wheats (Oda et al. 1980). Flour particle size parameters have been analyzed since the late 1980s by the laser-beam diffractometry method, and it has been shown that detailed flour particle size distributions could be easily determined using laser light apparatus (Devaux et al. 1998; Greffeuille et al. 2006). Flour particle size distribution is an important indicator of the quality of high-ratio flour (Posner and Hibbs 1997), and commercial wheat flour samples are often characterized according to their particle size properties (Gaines 1985; McDonald 1994). It would therefore be useful to determine the relationship between flour yield and flour particle size distribution (Wu et al. 1990; Fang and Campbell 2003; Kim et al. 2004) and, revealed the effect of median flour particle size $(\mu \mathrm{m})$ in wheat flour yield, so that the median flour particle size could be used as an index of flour yield in Japanese wheat breed programs, and developed a suitable method to evaluate flour yield of Japanese wheat (Nakamura 2014). Therefore, the aims of the current study are to investigate the differences of a median flour particle size between Japanese and Chinese common wheats, and could simply and quickly identify wheat genetic resources with high flour yielding in a systematic evaluation of N.A.R.O. institute of crop science in Japanese common wheat breeding program.

\section{Materials and Methods}

\section{Wheat samples}

The Chinese common wheat genetic resources obtained from National Agricultural Research Centre (NARC) in Tsukuba (Ibaraki, Japan) were used in this study. One hundred and seventy Chinese common wheat samples represented a wide range of hardness values in Chinese wheats. It has already revealed that the flour samples were classified into one of the three flour hardness categories based on their flour particle size distributions (Nakamura 2014). In order to compare with one hundred and sixty-five Japanese wheats (Nakamura 2014), one hundred and seventy Chinese common wheats were used for analyzing the median particle size distribution on wheat flour. In total, three hundred and thirty-five common wheat samples were used in this study.

\section{Test milling}

Fifty grams of cleaned wheat grains from each sample were milled according to the milling evaluation method of the Ministry of Agriculture and Forestry (1968). Briefly, there were 4 stages in the milling process; two breaking stages, one sizing stage and one reduction stage. This process produced a flour fraction, coarse and fine bran fractions, and also two short fractions. In the milling process each sample was tempered for 24 hours and then passed through a Brabender Jr. Quadrumat wheat flour laboratory mill (Brabender instrument Inc., Germany) at $25^{\circ} \mathrm{C}$ and $60 \%$ humidity. 


\section{Laser diffraction particle size analysis}

Two gram of flour were separated from each milled flour sample using a spinning riffle and placed in airtight vials. The flour particle-size distribution (PSD) of each $2 \mathrm{~g}$ sample was determined by a laser diffraction particle size analyzer, using a HELOS laser diffraction sensor fitted to a RODOS dry dispenser (Sympatec Ltd., Germany). The flour samples were dispersed (separated) at a constant temperature of $25^{\circ} \mathrm{C}$ and at $60 \%$ humidity. The dispersed flour particles were suspended in helium-neon gas. A standard-range laser analyzer with a $500 \mathrm{~mm}$ focal lens was used to generate different patterns of low-angle light scattering, and could measure particles ranging in diameter from 0.9 to $209.7 \mu \mathrm{m}$. The diffraction patterns were collected on a highly sensitive semi-circular multi-element detector. The PSDs were evaluated using the Mie theory, using software provided with the apparatus to estimate the proportions of each particle size as a function of the light intensity hitting the photo-detector. In addition to the PSD of each sample, the software also calculated both the mean and the median flour particle size of each sample. The median flour particle size shows that the flour particle size $(\mu \mathrm{m})$ of fifty percentage diameter under calculation in a particle analyzer, it is different from the mean flour particle size $(\mu \mathrm{m})$ in all flour.

\section{Categorization of wheat flour hardness after test milling}

The flour samples were classified into one of the three flour hardness categories based on their flour particle size distributions (Nakamura 2014). Samples were classified as soft, medium-soft or hard type, depending on whether they matched flour particle size distribution patterns I, II or III, respectively (Fig. 1 and Table 1). The soft, medium-soft and hard

Table 1. Median flour particle size (diameter) range and number of Chinese and Japanese common wheat varieties categorized in flour particle size distribution patterns I, II, III and number of varieties in each category

\begin{tabular}{|c|c|c|c|c|}
\hline $\begin{array}{l}\text { Flour particle size distribution pattern } \\
\text { groups }\end{array}$ & Pattern I & Pattern II & Pattern III & \multirow[t]{2}{*}{ Total } \\
\hline Wheat types & (soft) & (medium-soft) & (hard) & \\
\hline \multicolumn{5}{|l|}{ Chinese } \\
\hline Median flour particle range $(\mu \mathrm{m})$ & $30.62 \sim 36.87$ & $36.27 \sim 80.76$ & $67.67 \sim 100.89$ & \\
\hline Number of samples & 6 & 88 & 76 & 170 \\
\hline Percentage of total & $3.5 \%$ & $51.8 \%$ & $44.7 \%$ & $100 \%$ \\
\hline \multicolumn{5}{|l|}{ Japanese } \\
\hline Median flour particle range $(\mu \mathrm{m})$ & $26.83 \sim 37.60$ & $35.66 \sim 71.32$ & $66.92 \sim 93.56$ & \\
\hline Number of samples & 42 & 90 & 33 & 165 \\
\hline Percentage of total & $25.5 \%$ & $54.6 \%$ & $20.0 \%$ & $100 \%$ \\
\hline
\end{tabular}

Pattern I is soft wheat type, pattern II is medium-soft wheat type, and pattern III is hard wheat type, respectively. Japanese wheat data is from Nakamura (2014), for comparison with the Chinese data in this study. 


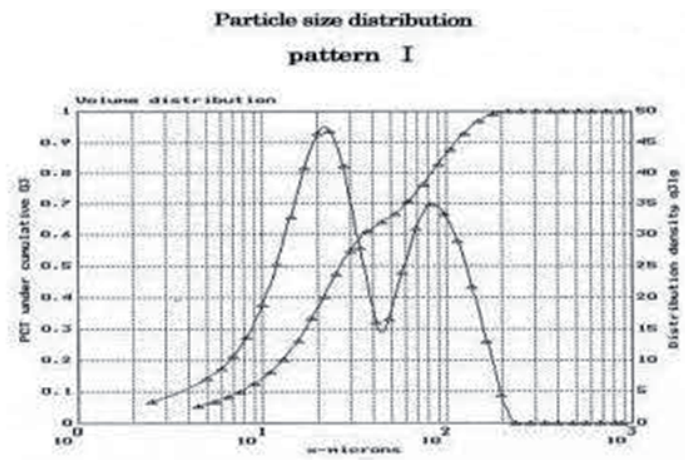

Particle aize distribution

pattern II

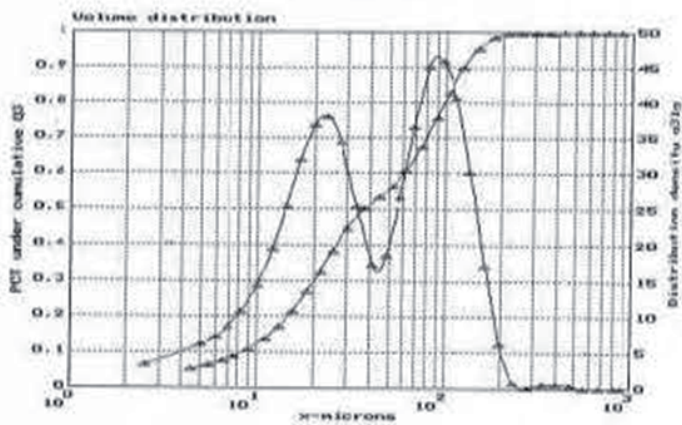

Particle aize diatribution

pattern III

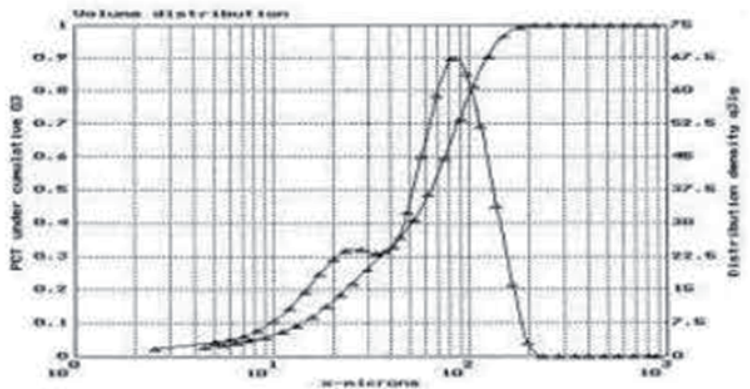

Figure 1. Representative flour particle size distribution patterns of common wheat varieties Pattern I soft type wheat; Pattern II medium-soft type wheat; Pattern III hard type wheat 
wheats could be clearly separated by particle size distribution analysis (Fig. 1), indicating that; flour particle size distribution depended on flour hardness. As the flour particle size distributions were either unimodal or bimodal, the standard deviation was irrelevant. Synthetic parameters relating to the flour particle size distributions could be obtained by using multiple regression analysis.

\section{Results}

Table 1 shows that the 170 Chinese common wheat varieties could be categorized into three flour particle size distribution pattern groups, corresponding to "soft", "mediumsoft" and "hard" wheat types, respectively. Representative examples of each pattern are shown in Fig. 1; Patterns I and II represent the typical bimodal particle size distributions of Chinese soft and medium-soft wheats. In my previous research on Japanese varieties, it was showed that varieties with soft wheat particle size distributions were associated with low flour yield (Nakamura 2014). The main peak in particle size occurred at around $20 \sim 30 \mu \mathrm{m}$ diameter, with a second smaller population around $80 \sim 90 \mu \mathrm{m}$ diameter.

In the previous study on Japanese wheats, multiple regression analysis showed that flour yield (Y) was significantly related to median flour particle size (X1) and mean flour particle size $(\mathrm{X} 2)$, according to the formula $\mathrm{Y}=66.03+0.32 \mathrm{X} 1-0.16 \mathrm{X} 2(\mathrm{R}=0.75)$ (Nakamura 2014). However, the same study also showed a very high correlation between median and mean flour particle size $(\mathrm{r}=0.97)$. Based on the regression equation and the high correlation, the analysis showed that the most important factor for determining flour yield was median flour particle size. Therefore, in this current study, the median flour particle size of the 170 Chinese wheats was focused, to identify the Chinese wheat genetic resources with good flour milling quality, and to compare the Chinese median flour particle size data with that of the 165 previously analyzed Japanese wheats.

Table 1 indicates that a median flour particle size (diameter) range and number of 170 Chinese, and 165 Japanese common wheat varieties for comparing to Chinese data (Nakamura 2014), respectively. Median flour particle size range is widely distributed in Chinese wheats, the same as Japanese wheats. However, there were significant differences the median flour particle size distribution between the Chinese and Japanese wheats. Therefore, it was revealed that flour particle size distribution pattern III hard type was major wheat type in Chinese wheat, it was different from Japanese wheats. In the analysis of the 170 Chinese varieties, only 6 varieties were categorized as Pattern I "soft" wheat with a median flour particle size of $30.62 \mu \mathrm{m} \sim 36.87 \mu \mathrm{m}, 88$ varieties were categorized as Pattern II "medium-soft" wheat with a median flour particle size of $36.27 \mu \mathrm{m} \sim 80.76$ $\mu \mathrm{m}$, and 76 varieties were categorized as Pattern III "hard wheat" with a median flour particle size of $67.67 \mu \mathrm{m} \sim 100.89 \mu \mathrm{m}$ (Table 1). These results indicated that nearly $45 \%$ of Chinese wheats were classified as hard wheat, on the other hand, only about $4 \%$ were classified as soft wheat.

In Fig. 2, there was a wide variation in the median particle size of the 170 Chinese wheat varieties, ranging from $30.62 \mu \mathrm{m}$ for a soft variety to $100.89 \mu \mathrm{m}$ for a hard variety. However, only one variety had a median flour particle size of more than $100 \mu \mathrm{m}$, which 
is typically associated with a higher flour yield. Hard bread wheats typically have a median flour particle size of more than $100 \mu \mathrm{m}$ and a large proportion have a hard particle distribution pattern (Devaux et al. 1998).

In Table 2, seven Japanese varieties possessed a median flour particle size of less than $30 \mu \mathrm{m}$ diameter, no Chinese wheats possessed it. Fifty-four Japanese wheat possessed a median flour particle size of $30 \sim 40 \mu \mathrm{m}$, and 43 Japanese wheats possessed a median flour particle size of $40 \sim 50 \mu \mathrm{m}$. That was more than fifty-eight percent of the Japanese wheats, and a median flour particle size of $30 \sim 40 \mu \mathrm{m}$ was the most Japanese wheat flour particle size distribution. On the other hand, a median flour particle size of $30 \sim 40 \mu \mathrm{m}$ was the minor Chinese wheat flour particle size. In hard wheat type, a median flour particle size of $80 \sim 90 \mu \mathrm{m}$ was the most Chinese wheat flour particle size distribution. Eleven varieties had a median flour particle size of more than $90 \mu \mathrm{m}$, which is typically associated with a higher flour yield. Eighty-six Chinese varieties (more than fifty percentage) had a median flour particle size of more than $70 \mu \mathrm{m}$, on the other hand, only 33 Japanese varieties (only twenty percentage) had the median flour particle size $>70 \mu \mathrm{m}$. In Chinese wheats, hard type wheat in a median flour particle size was major type, and soft type wheat was minor.

\section{Discussion}

To investigate a genetic resource for developing a higher flour yielding variety in Japanese wheat breeding, the flour yield of one hundred and seventy samples in Chinese common wheat were investigated in relation to the median flour particle size and the flour particle size distribution patterns as determined by laser diffraction in this study. In the previous study, it has already showed that the most important factor in a milling test or determining flour yield was a median flour particle size (Nakamura 2014). In that study, flour yield was most strongly associated with the median flour particle size in the flour samples. These results indicate the potential to develop a flour yield evaluation method in Chinese wheat varieties using laser diffraction methods, and that median flour particle size analysis could be a useful indicator of Chinese wheat flour quality for wheat breeding.

Wheat flour yield is the most important technical and economic factor with regard to flour milling, and plays an important part in the buying decisions of many milling companies. Experimental and laboratory milling determines wheat milling quality, and therefore has received much attention from wheat breeders and flour millers. The soft wheat breeding strategy in Japan is aimed at selecting the varieties with a good udon-noodle making quality, and a higher flour yield which also can have a major effect on the quality of noodle products. The most important aspect of wheat quality is a higher flour yield at milling, in order to satisfy market demands in Japan (Yamashita 1994). It is well known that hard and soft wheat varieties differ in flour yield after milling. Therefore, it is important to develop methods to quickly and accurately determine flour yields of new potential genetic resources for wheat breeding programs. The merit of a Brabender Jr. Quadrumat mill is that it is very easy to operate and gives good reproducible results, and therefore it 
could be used to evaluate common wheats with respect to differences in flour milling quality. These results from the milled flour analysis of the Chinese wheat samples in this study indicate that the median flour particle size could be a useful tool for selecting common wheat lines with a higher flour yield, such as the F4 early generation. Modern electric and computing technology (i.e. particle dispensing and laser diffraction equipment and analysis software) have made it possible to analyze flour particle size distribution more quickly and with more reproducible results than with other methods such as sieve analysis. In this study, Chinese wheat genetic resources with high flour milling yield could be quickly identified in a systematic evaluation of N.A.R.O. materials, and enabling suitable crosses to be subsequently made with high grain yielding varieties in the N.A.R.O. breeding system. For example, by using wheat flour particle size distribution analysis, high flour yielding materials have been selected from Chinese and Japanese wheat germplasm, lines and varieties for breeding higher flour yielding udon-wheat lines. Also, 51 common wheat lines (named Kannon lines No.1-No.51) have been bred from crossing selected Japanese and Chinese breeding materials with high flour yielding/Japanese udon-wheat varieties with high grain yields and good udon-noodle making quality.

Since judging milling texture by the appearance of wheat grains is often misleading (Yamashita 1994), flour particle size distribution analysis could play an important role in the Japanese wheat breeding program. In general, udon-wheats give a lower flour extraction rates, whereas ASW and/or WW standards have a higher flour extraction rates, and this is an important economic issue for Japanese milling companies (Yamashita 1994). Previously, the flour hardness index has generally been determined based on the glassy kernel ratio in wheat flour (Ministry of Agriculture and Forestry 1968; Nakamura et al. 1990). However, this method cannot be used to rapidly evaluate the flour yield as part of the wheat-breeding program (Nakamura and Fujimaki 2002). Therefore, a milling evaluation index was developed for Japanese wheat varieties, together with a method to predict the flour yield using laser diffraction, which is a new technology for Japanese wheat research, instead of using the glassy kernel ratio evaluation (Nakamura 2014). The flour particle size distribution analysis, especially using the median flour particle size could be used in common wheat breeding programs to rapidly and reliably evaluate and predict flour yield. In addition, flour particle size is a heritable trait in soft wheat varieties and is not greatly affected by crop year (Donelson and Yamazaki 1972). Therefore, median flour particle size is a genotypic fingerprint that can be used for many purposes, including hard and/or soft wheat variety protection, registration, certification, and crossing, as well as functioning as a tool in wheat breeding. The median flour particle size analysis technique, used in this study and previous study to select varieties suitable for noodle production in Japanese wheat breeding and to evaluate flour quality, is easy to perform and does not require expensive equipment. Although, the median flour particle size might not be the primary factor for determining noodle-making quality, it might be linked to other parameters that contribute to wheat quality and yield. Wheat breeders who want to breed medium-soft udon-wheat lines with higher flour yield should make more use of the median flour particle size to more easily and rapidly select suitable materials from the Japanese and Chinese common wheat germplasm, lines, and varieties. 


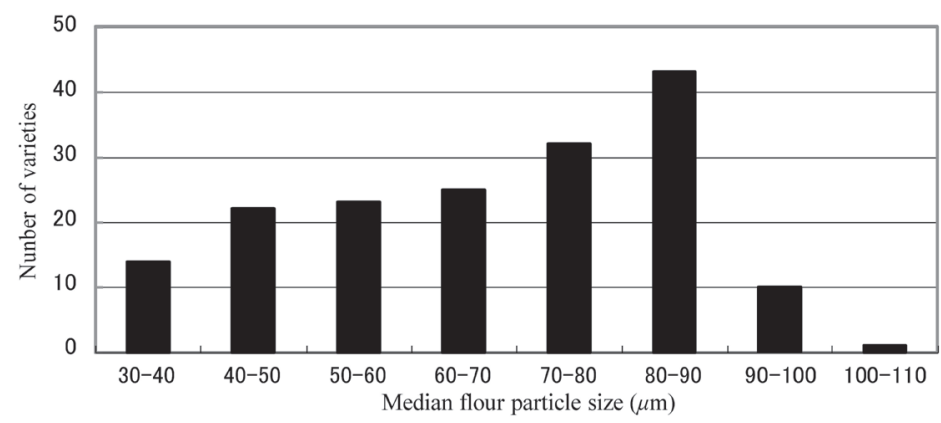

Figure 2. Median flour particle size distribution in 170 Chinese common wheats

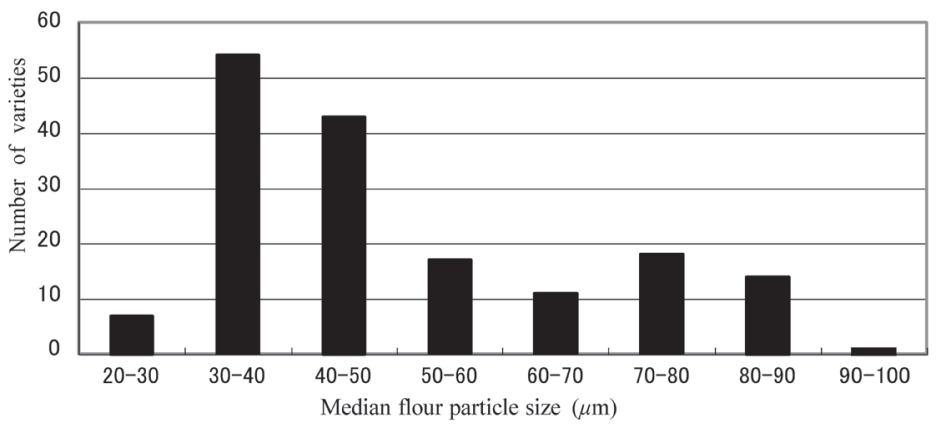

Figure 3. Median flour particle size distribution in 165 Japanese common wheat (cited by Nakamura (2014) for comparing to Chinese data)

The Japanese variety 'Norin No. 61' was used as a control in the evaluation of the udon-noodle making quality of the other wheat varieties, because 'Norin No. 61' is generally known as one of leading varieties for udon-noodle products (Oda et al. 1980). The pattern II flour particle size distribution is also found in overseas varieties such as Australian standard white (ASW) and Western white standards (WW) (Nakamura unpublished), indicating a soft wheat milling behavior with a higher flour yield. Also, a major strategy to improve Japanese wheat production is to develop higher flour yielding varieties similar to ASW and WW (Yamashita 1994). The hard wheat variety flour particle size distribution pattern III exhibited only one main peak mode around $80 \sim 100 \mu \mathrm{m}$ diameter. The main difference between the hard wheat pattern and the soft and medium-soft wheat patterns was the much lower proportion of smaller particle sizes in the hard wheat. These distribution patterns indicate that typically the median and mean flour particle sizes of soft wheats are lower than those of hard wheats. The greater proportions of smaller particles in soft and medium-soft wheats is due to the fact that the milling process produces more isolated starch granules in these two types of wheat than in the hard wheats. 
Table 2. Frequency distributions for median flour particle size (diameter) ranges of Chinese and Japanese common wheat varieties

\begin{tabular}{|l|c|c|c|c|c|c|c|c|c|c|}
\hline $\begin{array}{c}\text { Median flour } \\
\text { particle range }(\mu \mathrm{m})\end{array}$ & $20 \sim 30$ & $30 \sim 40$ & $40 \sim 50$ & $50 \sim 60$ & $60 \sim 70$ & $70 \sim 80$ & $80 \sim 90$ & $90 \sim 100$ & $100 \sim 110$ & Total \\
\hline Chinese & 0 & 14 & 22 & 23 & 25 & 32 & 43 & 10 & 1 & 170 \\
\hline Japanese & 7 & 54 & 43 & 17 & 11 & 18 & 14 & 1 & 0 & 165 \\
\hline
\end{tabular}

A major strategy in the Japanese wheat breeding program is to improve the low flour yield of 'Norin No. 61' which has good udon-noodle making quality (Yamashita 1994). It is known that the flour fraction $<35 \mu \mathrm{m}$ is composed mainly of isolated starch granules and that soft-wheat flour has a large proportion of such starch granules. Devaux et al. (1998) reported that particles around the $25 \mu \mathrm{m}$ mode correspond mainly to isolated starch granules, and that the main difference between hard and soft wheat flours is in the proportion of isolated starch granules freed when the grains are fragmented during the flour milling process. Starch quality characteristics are very important for udon-noodle making-quality (Oda et al. 1980). Also, flour with high damage starch to granules and also small flour particle size require more water absorption during noodle-making (Oh et al. 1985). Therefore, the flour particles around $20 \sim 35 \mu \mathrm{m}$ in udon-noodle wheats appear to play an important role in good noodle-making quality. Since udon-noodles are made of soft wheat flour and not hard bread flour, the flour yield of udon-wheats is relatively low, and the low flour yield of 'Norin No. 61' did not satisfy milling market demands in Japan.

The analysis of the Chinese wheats in this study, and comparison with the previously analyzed Japanese wheats (Nakamura 2014) showed clear differences in the median particle sizes of the Chinese and Japanese wheats (Figs 2 and 3, Tables 1 and 2). There were far fewer soft type Chinese wheats and far more hard type wheats than in the analysis of the Japanese wheat samples. It is believed that cultivated common wheat originated in the Middle and Near East traveled via the Silk Road through China to the Far East and Japan. Therefore, Japan is a remote region in the world for common wheat production. In the course of its long journey and its adaptation to diverse local environments, Japanese common wheat seems to have lost its genetic diversity such as hard wheat type. It has already reported that Japanese common wheat has developed a unique composition of Glu-1 glutenin alleles and a narrow common wheat genetic base, based on a consequence of its spread, adaptation, and phenotypic refinement (Nakamura 2008). The variability released by both environmental and genetic factors could easily lead to different subpopulations of the founders. This in turn, could lead to the establishment of more than one reproductively isolated population from a single founder through population genetic mechanisms for common wheat. Each bottleneck was followed by a flush of rapid population growth, so once again there were optimal conditions for inducing genetic change (Templeton 1980). With this design from China, the common wheat was exposed to a selective bottleneck induced by the external environment, as well as a founder effect (since all populations went through a narrow bottleneck). Consequently, the selective bottleneck was extremely intense and, in fact, most ancestral populations may be become extinct in Japan 
(Nakamura and Fujimaki 2002). Therefore, the result in this study may be explained either by the founder effect or by a selective bottleneck effect in Japanese common wheat genetic resources.

In conclusion, a median flour particle size distribution analysis is a simple but effective and useful analytical tool for measuring and selecting the good flour milling quality in Chinese and Japanese wheat genetic resources. To further improve flour milling quality and develop unique grains of common wheat in Japan, the genetic base of domestic wheat will have to be broadened. The ease with which genetic changes occur in Japan strongly implies a lack of genetic variability in natural populations of Japanese common wheats. The theory presented here, the founder effect and/or selective bottleneck effect can help explain the wheat genetic revolution model of speciation and, more importantly, has generated testable predictions that can be examined in both the natural and artificial selection using current methodologies and systems. Therefore, it is hoped that this theory of Japanese common wheat helps to demonstrate how population genetic theory can be applied to the problem of speciation in more extensive and through fashion than it has in the past.

\section{References}

Devaux, M.F., Monredon, F.D., Guibert, D., Novales, B., Abecassis, J. 1998. Particle size distribution of break, sizing and middling wheat flours by laser diffraction. J. of Sci. Food Agric. 78:237-244.

Donelson, D.H., Yamazaki, W.T. 1972. Soft wheat flour particle-size analysis by integrated sieve and coulter counter procedures. Cereal Chem. 49:641-648.

Fang, C., Campbell, G.M. 2003. On predicting roller milling performance: Effect of roll disposition on the particle size distribution from first break milling of wheat. Cereal Sci. 37:21-29.

Gaines, C.S. 1985. Association among soft wheat flour particle size, protein content, chlorine response, kernel hardness, milling quality, white layer cake volume, and sugar-snap coolie spread. Cereal Chem. 62:290292.

Greffeuille, V., Abecassis, J., Rousset, M., Oury, F.X., Faye, A., L' Helgouach, C.B., Lullien-Pellerin, V. 2006. Grain characterization and milling behaviour of near-isogenic lines differing by hardness. Theor. Appl. Genet. 114:1-12.

Ikeda, R. 1961. Research on Japanese hard wheat. Bulletin of the Tokai-Kinki National Agricultural Experimental Station. pp. 1-55. (In Japanese)

Kim, W.C., Kerr, S.G., Jonson, W.L., Gaines, C.S. 2004. Effect of heating temperature on particle size distribution in hard and soft wheat flour. J. Cereal Sci. 40:9-16.

McDonald, C.E. 1994. Collaborative study on particle size in wheat flour by laser instrument. Cereal Food World 39:29-33.

Ministry of Agriculture and Forestry 1968. The evaluation method for Japanese hexaploid wheat. Tokyo, Japan, pp. 10-26. (in Japanese)

Nakamura, H. 2014. Effect of median flour particle size on flour yield in Japanese common wheats. Cereal Res. Commun. 42:668-676.

Nakamura, H. 2008. Possible transmission route for common wheat to the Far East in Asia. Crop Sci. 48:11171123.

Nakamura, H., Fujimaki, H. 2002. Specific Glu-D1f allele frequency of Japanese common wheat compared with distribution of $G l u-1$ alleles in Chinese wheat. Cereal Chem. 79:486-490.

Nakamura, H., Sasaki, H., Hirano, H., Yamashita, A. 1990. A high molecular weight subunit of wheat glutenin seed storage protein correlates with its flour quality. Japanese J. of Breed. 40:485-494.

Oda, M., Yasuda, Y., Okazaki, S., Yamauchi, Y., Yokokawa, Y. 1980. A method of flour quality assessment for Japanese noodles. Cereal Chem. 57:253-254. 
Oh, N.H., Seib, P.A., Ward, A.B., Deyoe, C.W. 1985. Noodles. IV. Influence of flour protein, extraction rate, particle size, and starch damage on the quality characteristics of dry noodles. Cereal Chem. 62:441-446.

Posner, E.S., Hibbs, A.N. 1997. Wheat Flour Milling. American Association of Cereal Chemist, St. Paul, MN, USA. pp. 219-220.

Templeton, A.R. 1980. The theory of speciation via the founder principle. Genetics 94:1011-1038.

Wu, Y.V., Stringfellow, A.C., Bietz, J.A. 1990. Relation of wheat hardness to air-classification yields and flour particle size distribution. Cereal Chem. 67:421-427.

Yamashita, A. 1994. Discussion of the Japanese hexaploid commercial wheats (Triticum aestivum). Nobunkyo, Publishing Ltd., Tokyo, Japan, pp. 1-71. (in Japanese) 